\title{
Accidental Dislodgement of Separated Instrument in the Maxillary Sinus: A Unusual Case
}

\section{Avisha Agrawal*, Pratima Shenoi, Rajesh Kubde, Snehal Sonarkar, Gautam Badole, Vandana Kokane, Ankita Ramteke and Purabi Edbor}

Department of Conservative Dentistry and Endodontics, VSPM Dental College,

Nagpur, India

*Corresponding Author: Avisha Agrawal, Department of Conservative Dentistry and Endodontics, VSPM Dental College, Nagpur, India.
Received: September 30, 2020

Published: October 28, 2020

(C) All rights are reserved by Avisha Agrawal., et al.

\begin{abstract}
Root canal therapy consists of a cascade of scientifically-based technical procedures. Failure to follow the standard protocols and lack of knowledge can lead to a number of iatrogenic accidents which are collectively called "endodontic mishaps". One of the most frequently encountered endodontic mishap is a separation of the endodontic instrument. Heroic attempts to remove separated instrument may lead to dislodgment of instrument in the periapical area. At times such instrument get dislodged in the maxillary sinus when treating upper maxillary molars and canines. Separated instrument in maxillary sinus, irrespective of their origin or nature, act as foreign body and Such objects should normally be removed at the earliest in order to minimize the likelihood of maxillary sinus complications. Studies have shown that some of the objects can cause chronic sinusitis because of the invasion of the sinus by highly virulent bacteria, while some remains asymptomatic. It is an uncommon finding to observe, an asymptomatic dental endodontic instrument in a position so close to the maxillary sinus without any oro-antral fistula formation and/or sinusitis attacks. This paper presents the case of a separated endodontic instrument which was pushed inadvertently in the maxillary sinus of a 41-year-old female patient.
\end{abstract}

Keywords: CBCT; Endodontic Instrument; Root Canal Treatment; Separation

\section{Introduction}

Endodontic therapy eliminates pulpal disease by a total debridement of diseased tissue. This helps in relieving the signs and symptoms of inflamed tissue [1]. To achieve this objective endodontic instruments medicaments and irrigants are used [2]. However, this instrument should remain within the confines of root canal system without extending periapically [3]. Because of anatomical variations of the canal and cyclic fatigue to endodontic instruments, they separate, which leads to mishap. This endodontic mishap are unavoidable during endodontic therapy [4]. Few studies have reported the prevalence of instrument separation during endodontic treatment is $1.83 \%$ [5]. The rate of separation of Nickel-Titanium (NiTi) rotary instruments are more than Stainless-steel within the range of $1.3 \%$ and $10 \%$ [6]. An adequate experience is necessary by the operator which can minimize the incidence of instrument separation [7]. In an endodontic mishap, such as instrument separated apically or overextended obturating material, may be associated with the migration of foreign bodies in the sinus [8].

Maxillary sinus is the largest paranasal air sinus with threewalled pyramid structure, situated bilaterally in the upper jaw, in relation to the maxillary posterior teeth [9]. The roots of the maxillary teeth are in close association with it, hence the knowledge of its anatomy is paramount for successful and uneventful endodontic treatment [10]. A foreign body reaction occurs on the connective tissue of the sinus membrane when there is an extrusion of the broken instrument and filling material into the sinus [10]. Some of the objects can cause chronic sinusitis because of the invasion of the sinus by highly virulent bacteria, while some do not cause 
any reaction [11]. Foreign bodies should be removed immediately from the paranasal sinuses, because although they may remain asymptomatic for a long period, eventually may lead to other complication such as sinusitis [12]. Some objects which are displaced into maxillary sinus may require surgical removal while some may be expelled spontaneously [12]. A case was reported in which a relatively heavy metallic objects, located in the maxillary antrum migrated to the sinus ostia against gravitational force, by the aid of mucociliary action, and was subsequently expelled from the nasal cavity [13].

In this case report, 10-k file was separated in the canal during endodontic treatment, while retrieving it was pushed into the sinus, eventually was then extruded from the maxillary sinus. This case report has been made accordingly to the recently updated PRICE guidelines by Nagendrababu., et al [14].

\section{Case Report}

A 41year old female patient reported to the Department with chief complaint of pain in upper left back region of jaw since 1 week. Patient gave history of dull aching continuous pain, with no change in intensity while consumption of hot/cold food and beverages. Clinically, deep mesioocclusal caries was seen with 26 . Tooth was firm and showed slight tenderness on vertical percussion. Pulp vitality test and radiographic investigations were done for the affected region. Electric pulp test (Analytic Technology, Redmond, WA, USA) gave non-vital results which was further confirmed by performing cold test (Coltene/whaledent Inc., Mahwah, NJ, USA). Radiographically, deep mesioocclusal radiolucency involving pulp horn and periodontal ligament widening was seen in the apical region with 26. Differential diagnosis was made between Acute alveolar abscess and apical periodontitis. Correlating the clinical and radiographic findings a diagnosis of symptomatic irreversible pulpitis with symptomatic apical periodontitis was made with 26 . A treatment protocol of Endodontic therapy with prosthetic rehabilitation was achieved.

Local anaesthesia containing 2\% lidocaine 1:80,000 epinephrine (Lignox, Indoco remedies Ltd, India) was administered before the rubber dam isolation (Coltene/whaledent, Inc., Mahwah, NJ, USA). A high speed handpiece and sterilized round bur (BR40: Mani, Inc., Tochigi, Japan) were used for root canal access opening, and walls were modified using safe end cutting diamond point (EX24: Mani, Inc., Tochigi, Japan) The root canals were accessed

Citation: Avisha Agrawal., et al. "Accidental Dislodgement of Separated Instrument in the Maxillary Sinus: A Unusual Case". Acta Scientific Dental Sciences 4.11 (2020): 68-73. with size \#10 K-file (Mani, Inc., Tochigi, Japan) and initial apical gauging was done. The root canal was irrigated using copious amount of 5.25\% NaOCl (Prime Dental Products Pvt. Ltd., India) and normal saline. Working length was recorded using electronic apex locator (Root ZX; J Morita, Irvine, CA) and was confirmed with digital intraoral radiograph (Gendex, Vix Win Platinum; KaVO, Detroit, Michigan).

Shaping and cleaning was done using crown down technique. GG drills (Mani, Tochigi, Japan) were used for enlarging the canal orifice up to \#3. Hand instrumentation was initiated with kerr file (\#10). During instrumentation of distobuccal canal, there was separation of endodontic instrument (\#10-k file) that was confirmed radiographically. The procedural accident of separated instrument was informed to patient. The canals were prepared using ProTaper universal (Dentsply Maillefer, Ballaigues, Switzerland) till size F-1 in mesial canal and F-3 in the palatal canal. A due consent was taken from the patient for the removal of separated instrument from distobuccal canal.

Then, hand instrumentation was continued to bypass and retrieve the instrument. However, the fragment got dislodged in maxillary sinus while attempting the removal of separated instrument. This was confirmed by taking radiographic images and the endodontic procedure was terminated. The temporary dressing cavity (3M Envision school project Exchange, GmbH Dental Products, Neuss, Germany) was placed in the pulp chamber. Post-operative instructions were given as not to blow nose or sneeze with the closed mouth. Avoid eating of hard food items. Further the patient was instructed to Inhale steam with menthol or benzoin 6 hourly which will help in stimulating serous glands.

Patient was referred to the Department of otolaryngology in medical hospital attached to institute. Orally, Antibiotics (Amoxil $500 \mathrm{mg}$ TDS, Zydus Cadila, Amoxicillin for 5 days) and Analgesics (Advil 400 mg TDS, Advil, Ibuprofen for 5 days), chlorhexidine mouth wash (Hexidrin, Chlorhexidine gluconate 0.2\%, Nanz Med Science Pharma Ltd) and oxymetazoline nasal spray(Afrin Sinus, Oxymetazoline $0.05 \%$ BDS, Navajo Manufacturing) were prescribed. Patient was informed about the procedure for management of separated instrument and was recalled after 5 days.

On recall, it was observed that there were no signs and symptoms of infection in the sinus. CBCT scan was done of the concerned region which revealed no separated instrument in the sinus. Obturation was completed in the other 2 canals and the root canal 
orifices were sealed with PermaFlo ${ }^{\mathrm{TM}}$ Pink (Ultradent Products Inc, South Jordan, USA). The scan was repeated after 2 months and no separated instrument was located. The case was collaboratively discussed in the presence of an otolaryngologist and an endodontist. After complete discussion we came to a conclusion that the separated instrument could have been expelled through the nostrils.

The distobuccal canal was then obturated with white ProRoot MTA (Dentsply, Tulsa Dental, Johnson City, Tennessee, USA) creating an apical plug of $3 \mathrm{~mm}$. A sterile cotton pellet moistened with sterile water was placed over the canal orifice and the access cavity was sealed with Cavity. After $72 \mathrm{~h}$, the hard set of MTA was confirmed and the remainder of the root canal was obturated with gutta percha using backfill with warm vertical compaction technique followed by composite restoration (Filtek Z350 XT Universal Restorative, $3 \mathrm{M}$ India). The patient has been kept under observation with scheduled monthly visit.

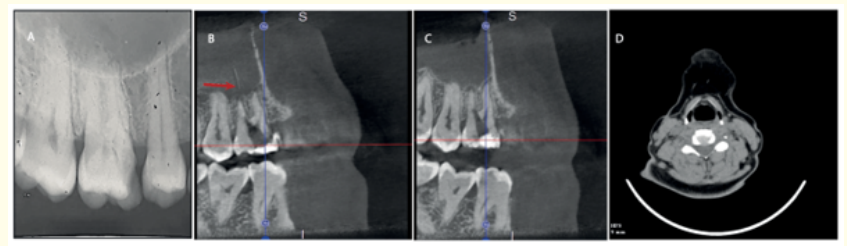

Figure 1: Separation of endodontic instrument (A) A preoperative radiograph that shows a deep mesioocclusal radiolucency involving pulp horn. (B) CBCT - Showing separated instrument in the maxillary sinus. (C) CBCT after 5 days showing disappearance of instrument in maxillary sinus. (D) CT scan image to confirm the movement of the instrument.

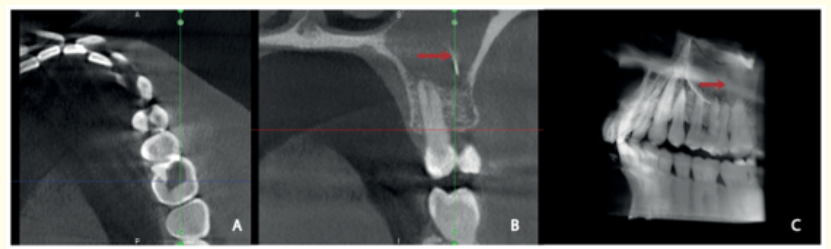

Figure 2: Different sections of the CBCT after file separation A. Axial section. B. Coronal section C. Sagittal section.

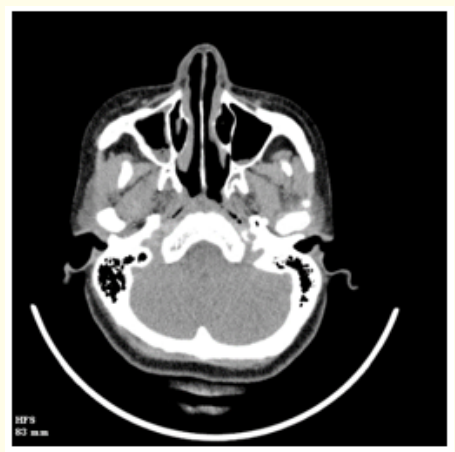

Figure 3: CT scan showing clear sinus.

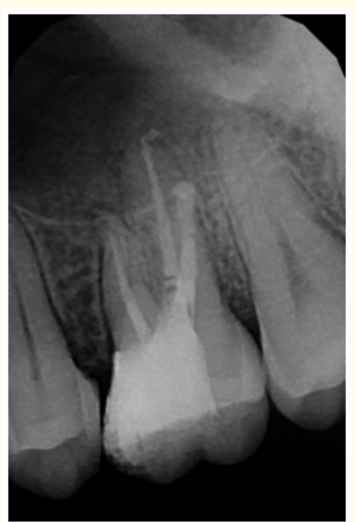

Figure 4: Post-operative radiograph.

\section{Discussion}

In clinical practice, one of the most frequently encountered endodontic mishap is separation of an endodontic instrument $[2,7,15,16]$. There is $19 \%$ reduction in healing and higher failure rates in cases with instrument separation [17]. These separations are related to several factors such as incorrect access opening, the curvature of the root canal, instrument fatigue, and the operator's experience [18].

A study reported that the buccal roots of maxillary molars have a higher incidence of instrument separation because of narrow roots [5]. It has been found that various biological factors and procedural errors have led the inability of endodontic material to be contained in the root canal system [19]. Any periapically extruded endodontic material can cause a foreign body reaction in the max-

Citation: Avisha Agrawal., et al. "Accidental Dislodgement of Separated Instrument in the Maxillary Sinus: A Unusual Case". Acta Scientific Dental Sciences 4.11 (2020): 68-73. 
illary sinus [19]. These reaction disturb the regular functioning of the system, as they do not respond well to treatment of rhinosinusitis [20]. Hence, It becomes imperative to retrieve the fragment which has gone beyond the apex.

Different foreign bodies which have been reported earlier with sinus perforation include endodontic materials [8], displaced fractured roots [21], dental impression materials [22], dental burs [23], sewing needles [24], amalgam fillings [25] and even dental implants [26]. Augusti., et al. reported $16 \%$ cases of foreign objects in the maxillary sinus. 10 out of 11 cases were found to be of odontogenic origin and 1 case of non- odontogenic origin [27].

In the present case, the endodontic instrument was separated in the middle third of distobuccal root of maxillary first molar while retrieving it was pushed into the apical third and then periapically into the maxillary sinus. To confirm the exact location of separated instrument 3-dimensional CBCT was used as it is considered as a very useful investigation tool for diagnosis of endodontic problems [19]. According to a study done by Brito., et al. CBCT images showed high values of accuracy in detecting separated endodontic instruments [28]. With regard to the present case, CBCT was able to discern separated instruments in the maxillary sinus in relation to distobuccal root.

Further, the obturation was completed in other two canals (mesiobuccal and palatal). These canals were sealed at the orifice opening using flowable composite. This was done to prevent any bacterial filtration if ever the restoration fails [29].

The CT scans were carried out as they produce a three-dimensional rendering to relevant anatomical structures in axial, sagittal and coronal planes. This provides a multiplanar high-resolution image that eliminates the superimposition of maxillary molars, allowing the clinician to distinguish between tissues of physical density. Whereas, maxillofacial CT scans allows for a detailed examination of the patient's paranasal sinus anatomy and detection of sinonasal inflammation, making this modality as the gold standard for radiographic evaluation in paranasal sinuses with chronic and recurrent acute sinonasal diseases [30]. In the present case, CT scan was done to check the movement of dislodged instrument to other parts.

Various case reports suggest that the objects from the maxillary sinus could be removed by using surgical and nonsurgical technique. These are endoscopic sinus surgery [31], Caldwell-Luc tech- nique [32] or combination of both [32], magnetic iron [20]. In this case non surgical treatment option was selected as the instrument was not seen in CBCT and CT scan. Similarly, other cases reported by Westermark., et al. [33] and Borgonovoa., et al. [34] showed removal of foreign objects from maxillary sinus non-surgically. The reason for this could be attributed to the position of antrum while sleeping is directed downwards which facilitates the foreign body towards natural ostium [35].

Killey and Kay stated that in lying position the negative pressure is created by inspiration hence position of the head and the size of the ostium is of a greater importance [36]. According to Bowditch, the ciliated epithelium is capable of $7 \mathrm{Gm}$.-mm. per minute per sq. $\mathrm{cm}$. of work [35]. Movement of the above object would be well within these limits. Once inside the ostium, the partial or complete obstruction produced would cause a rising intra-sinus pressure that in turn would hold the foreign body in place. Pressure atrophy would allow it to proceed gradually into the nose and hence be expelled [35]. It was then concluded that the file was pushed out from the sinus.

An $3 \mathrm{~mm}$ apical plug of MTA in distobuccal canal was created using hand condensation technique. Many studies have confirmed the superior biocompatibility of MTA as a root-end filling [37]. Remaining of the canal was then obturated with warm gutta percha using backfills as it provides a closer adaptation to dentinal walls and prevents any leakage [38]. One of the Limitations of this case report was, the dependency of the case on radiographs. Artifacts produced in the radiographs affects the accuracy of the diagnosis [39].

\section{Conclusion}

In contrast to most of the cases recorded in the literature, this case highlights the fact that all separated instrument into the maxillary sinus does not lead to mucosal reaction and sinusitis. Radiographic evaluation along with Cone beam computed tomography becomes the gold standard in evaluation of such foreign bodies. Interdisciplinary consultation between Radiologist and otolaryngologist is advisable for successful and long term prognosis of such similar cases. In cases where patient is asymptomatic and no further findings are reported, a more conservative approach such as patient observation/scheduled patient follow-ups to determine presence of any symptoms could be considered as an alternative for predictable management. 


\section{Acknowledgements}

The authors deny any conflict of interest related to this study.

\section{Bibliography}

1. Mandel E., et al. "Rotary Ni-Ti profile systems for preparing curved canals in resin blocks: Influence of operator on instrument breakage". International Endodontic Journal 32.6 (1999): 436-443.

2. Parashos P., et al. "Factors influencing defects of rotary nickeltitanium endodontic instruments after clinical use". Journal of Endodontics 30.10 (2004): 722-725.

3. Poveda R., et al. "Mental nerve paresthesia associated with endodontic paste within the mandibular canal: report of a case". Oral Surgery, Oral Med Oral Pathol Oral Radiol Endodontology 102.5 (2006): 46-49.

4. Torabinejad M. "Endodontic mishaps: etiology, prevention, and management". Alpha Omegan 83.4 (1990): 42-48.

5. Tzanetakis GN., et al. "Prevalence and Management of Instrument Fracture in the Postgraduate Endodontic Program at the Dental School of Athens: A Five-year Retrospective Clinical Study". Journal of Endodontics 34.6 (2008): 675-678.

6. Chatterjee K. "Search designs for searching for one among the two-and three-factor interaction effects in the general symmetric and asymmetric factorials". Annals of the Institute of Statistical Mathematics 42.4 (1990): 783-803.

7. Parashos P., et al. "Factors influencing defects of rotary nickeltitanium endodontic instruments after clinical use". Journal of Endodontics 30.10 (2004): 722-725.

8. Yamaguchi K., et al. "Gross extrusion of endodontic obturation materials into the maxillary sinus: a case report". Oral Surgery, Oral Medicine, Oral Pathology, and Oral Radiology 104.1 (2007): 131-134.

9. Wolf MK., et al. "Preoperative 3D imaging in maxillary sinus: Brief review of the literature and case report". Quintessence International 46.7 (2015): 627-631.

10. Kafas P., et al. "Dysaesthesia in the mental nerve distribution triggered by a foreign body: A case report". Cases Journal 2.10 (2009): 1-4.

11. Harrison K., et al. "Fixation tack penetration into the maxillary sinus: A case report of a guided bone regeneration procedure complication". American Journal of Case Reports 14 (2013): 4347.
12. Sohn DS., et al. "Removal of displaced foreign body from the maxillary sinus using replaceable bony windows and saline irrigation, followed by suctioning of the foreign body". Implant Dentistry 20.2 (2011): 112-117.

13. Pang KP., et al. "Migration of a foreign body in the maxillary sinus illustrating natural mucociliary action". Medical Journal of Malaysia 60.4 (2005): 523-525.

14. Nagendrababu V., et al. "PRICE 2020 guidelines for reporting case reports in Endodontics: a consensus-based development". International Endodontic Journal (2020): 619-626.

15. Grossman LI. "Guidelines for the prevention of fracture of root canal instruments". Oral Surgery, Oral Medicine, Oral Pathology, and Oral Radiology 28.5 (1969): 746-752.

16. Frota LMA., et al. "Removal of Separated Endodontic K-File with the Aid of Hypodermic Needle and Cyanoacrylate". Case Reports in Dentistry (2016).

17. Mantri S. "Management of fractured root canal treated mandibular molar with separated endodontic instrument extending in periapical region". SAGE Open Medical Case Reports 6 (2018): 2050313X1880925.

18. Brito-júnior M., et al. "Alternative Techniques to Remove Fractured Instrument Fragments from the Apical Third of Root Canals: Report of Two Cases 26 (2015): 79-85.

19. Brooks JK and Kleinman JW. "Retrieval of extensive gutta-percha extruded into the maxillary sinus: Use of 3-dimensional cone-beam computed tomography". Journal of Endodontics 39.9 (2013): 1189-1193.

20. Shao L., et al. "Removal of Maxillary Sinus Metallic Foreign Body Like a Hand Sewing Needle by Magnetic Iron". International Journal of Clinical Pediatric Dentistry 7.1 (2014): 61-64.

21. Barclay JK. "Root in the maxillary sinus". Oral Surgery, Oral Medicine, Oral Pathology, and Oral Radiology 64.2 (1987): 162164.

22. Winstock D and Warnakulasuriya S. "Impression material presenting in the maxillary antrum as a foreign body". British Dental Journal 160.2 (1986): 54-55.

23. Khoury F. "Burs and root tips in the maxillary sinus TT - Bohrer und Wurzelrest in der Kieferhöhle”. Quintessenz 36.7 (1985): 1233-1237.

24. Holmes A. "Sewing needle as an antral foreign body". British Dental Journal 162.4 (1987): 153-154. 
25. Burnham R and Bridle C. "Aspergillosis of the maxillary sinus secondary to a foreign body (amalgam) in the maxillary antrum". British Journal of Oral and Maxillofacial Surgery 47.4 (2009): 313-315.

26. Iida S., et al. "Migration of a dental implant into the maxillary sinus. A case report". International Journal of Oral and Maxillofacial Surgery 29.5 (2000): 358-359.

27. Bodet Agusti E., et al. "[Foreign bodies in maxillary sinus]". Acta Otorrinolaringologica Española 60.3 (2009): 190-193.

28. Ramos Brito AC., et al. "Detection of Fractured Endodontic Instruments in Root Canals: Comparison between Different Digital Radiography Systems and Cone-beam Computed Tomography". Journal of Endodontics 43.4 (2017): 544-549.

29. Jenkins S., et al. "Sealing ability of three materials in the orifice of root canal systems obturated with gutta-percha". Journal of Endodontics 32.3 (2016): 225-227.

30. Little RE., et al. "Odontogenic sinusitis: A review of the current literature". Laryngoscope Investigative Otolaryngology 3.2 (2018): 110-114.

31. Sgaramella N., et al. "Displacement of Dental Implants Into the Maxillary Sinus: A Retrospective Study of Twenty-One Patients". Clinical Implant Dentistry and Related Research 18.1 (2016): 62-72.

32. Chiapasco M., et al. "The management of complications following displacement of oral implants in the paranasal sinuses: a multicenter clinical report and proposed treatment protocols". International Journal of Oral and Maxillofacial Surgery 38.12 (2009): 1273-1278.

33. Westermark AH. "Spontaneous removal of foreign bodies from the maxillary sinus: Report of a case". Journal of Oral and Maxillofacial Surgery 47.1 (1989): 75-77.

34. Borgonovo A., et al. "Displacement of a dental implant into the maxillary sinus: case series". Minerva Stomatologica 59.1-2 (2010): 45-54.

35. Parker W. “In the maxillary sinus” (1953): 100-105.

36. Killey HC. "Possible sequelae when a tooth or root dislodged into the maxillary sinus". British Dental Journal 116 (1964): 73-77.

37. Asgary S and Ehsani S. "MTA resorption and periradicular healing in an open-apex incisor: A case report". Saudi Dental Journal 24.1 (2012): 55-59.
38. Wu MK., et al. "Fluid transport along gutta-percha backfills with and without sealer". Oral Surgery, Oral Medicine, Oral Pathology, and Oral Radiology 97.2 (2004): 257-262.

39. Angelopoulos C., et al. "A Comparison of Maxillofacial CBCT and Medical CT". Atlas of the Oral and Maxillofacial Surgery Clinics of North America 20.1 (2012): 1-17.

\section{Assets from publication with us}

- Prompt Acknowledgement after receiving the article

- Thorough Double blinded peer review

- Rapid Publication

- Issue of Publication Certificate

- High visibility of your Published work

Website: www.actascientific.com/

Submit Article: www.actascientific.com/submission.php Email us: editor@actascientific.com

Contact uS: +919182824667 